Digital Press Social Sciences and Humanities

L'échange de pouvoir : un reflet de la vie carcérale dans le film Un prophète de Jacques Audiard

Damar Jinanto

Proceeding of Conférence internationale sur le français 2018

Joesana Tjahjani, Merry Andriani, Sajarwa, Wening Udasmoro (eds) 


\title{
L'échange de pouvoir : un reflet de la vie carcérale dans le film Un prophète de Jacques Audiard
}

\author{
Damar Jinanto \\ Universitas Indonesia, Depok, Indonesia \\ *e-mail : jirojinanto@gmail.com
}

\section{Résumé}

Considérant un film comme réflexion d'une pratique sociale, il soulève généralement le thème social. Dans ce contexte, le film est un instrument pour communiquer les problèmes dans la société. Le thème du pouvoir dans un espace social particulier se présente dans Un prophète, réalisé par Jacques Audiard. Ce film raconte l'histoire du personnage principal, Malik El-Djebena, qui a été détenu en prison pendant 6 ans. Dans la prison, El-Djebena, faisant partie du groupe arabe se trouve face à César Luciani, leader du groupe corse. Il est obligé de trouver une stratégie pour lutter contre César. Cet article parle de la relation de pouvoir des personnages à travers les aspects narratifs et cinématographiques du film, ainsi que le concept du pouvoir de Pierre Bourdieu. Il indique la détermination de la position des personnages du film en face du pouvoir dépendant de la défense des capitaux dans un espace social comme une prison. Le concept de Bourdieu montre également la forme de l'échange de pouvoir qui se passe parmi eux. Ce changement de position de pouvoir a des relations avec la propriété des capitaux de chaque personnage. Les résultats de l'analyse montrent le système de pouvoir aux personnages et à l'espace social par rapport à l'accumulation et la rétention des capitaux détenus en prison. La possession des capitaux provoque le changement de positions d'un personnage dans ce milieu particulier. Le texte désigne les efforts des personnages à obtenir les capitaux qui sont nécessaires dans l'échange de pouvoir.

\section{Mots-clés}

pouvoir, capital, espace social, Un prophète

\begin{abstract}
Considering that film is a form of social practice, it mostly brings social topic to be discussed. In this context, a film is a tool to talk about the problems within the society. Topic about power relation in certain social arena is present in Un prophète (The Prophet), directed by Jacques Audiard. This film tells a story about Malik El-Diebena living behind the bars for six years. In the prison, El-Diebena, who is the part of Arabian community, has had conflict with César Luciani, the leader of Corsican community. He had to find a strategy to fight against César. This article talks about power relation among the characters to fight against social structure based on the narrative and cinematographic aspects by using the concept of power relation by Pierre Bourdieu. The film provides the social hierarchy of the characters based on the capitals that apply in the prison as social arena. Bourdieu's concept also shows the power exchange between them. This interaction depends on the amounts of the capitals that each characters have. The result explains the power system between the characters and the social arena regarding the capital accumulation hold in prison. The social capitals lead to the position shifts in this arena. The text portrays the characters' efforts to obtain the capitals that are necessaries in the power exchange.
\end{abstract}

\section{Keywords}

power, capital, social arena, a prophet 


\section{Introduction}

En comprenant un film comme un moyen d'expression artistique, Boggs et Petrie dit que le film est principalement similaire à d'autres médias artistiques en communiquant à travers des images et des sons (p. 3). Ils affirment que l'analyse de film nécessite une observation attentive des images, des sons et des mouvements qui se produisent à l'écran. En observant le film, tout le monde donnera une grande appréciation. Par conséquent, l'appréciation peut aussi évoquer une compréhension approfondie du film (p. 5-9). On peut supposer que les récompenses des festivals de film sont la réaction des observateurs de film qui sont capables d'analyser des films grâce à la compréhension du film.

En France, Jacques Audiard est déjà connu comme un réalisateur qui reçoit souvent des prix dans plusieurs festivals de film prestigieux, tels que le César et le Festival de Cannes. Un prophète est l'un des films de Jacques Audiard qui a remporté plusieurs prix prestigieux. Le plus grand succès de ce film c'est l'obtention beaucoup de trophées de César, récompenses cinématographiques spécifiques aux films français. Un prophète, le cinquième film de Jacques Audiard dont durée d'environ 140 minutes, a toujours le même genre aux autres films d'Audiard. En général, tous les films réalisés par Audiard ont un genre dramatique. Cependant, chaque drame présenté par Audiard comporte des éléments de crime et de suspense.

Un prophète raconte l'histoire de Malik El-Djebena (interprété par Tahar Rahim), un garçon de 19 ans qui a été détenu pendant six ans dans la prison de La Centrale. Malik, en tant que nouveau prisonnier d'origine arabe, a toujours suivi les ordres donnés par César Luciani (interprété par Niels Arestrup), le chef du groupe corse, afin que le groupe arabe en prison considère Malik comme un membre du groupe corse. Le début de la rencontre de Malik avec César a eu lieu lorsque César lui a ordonné de tuer Reyeb. Après que Malik ait réussi à tuer Reyeb, Malik a été baptisé en tant que subordonné de César, surtout après que la plupart des membres du groupe corse aient été libérés de prison. Malik était chargé pour les missions de César, voire des tâches en dehors de la prison. César a cherché un moyen pour Malik d'obtenir la permission de sortir de la prison pour une journée.

César a perdu son pouvoir à la fin du film. Malik, étant contraint d'obéir à la domination de César, a finalement réussi à le faire tomber. Les batailles de Malik et César se comprennent par la lutte de la position de pouvoir. La détermination de la " victoire » ou de la " perte », cela dépend de la défense ou de la distribution du capital des personnages dans un espace social (Widjojo, p. 45). Les relations entre la propriété du capital et les luttes de pouvoir parmi les personnages peuvent être étudiées plus profondément pour voir la position dominante dans des champs de combat.

La pratique de la domination découle de la situation, du capital et de la stratégie des personnages, de sorte que la représentation graphique des relations de repose sur la propriété et la composition du capital des personnages (Haryatmoko, p. 16). Cette propriété du capital est le facteur principal pour déterminer un personnage qui est capable de dominer les autres. On a appris que chaque personnage est en train d'essayer d'obtenir ou de conserver le capital dont il possède.

Cette théorie peut être appliquée pour voir les luttes de pouvoir entre les personnages dans Un prophète. Chaque personnage essaie de développer ou de maintenir un capital. Parallèlement au développement du capital de caractère, le pouvoir qui s'y produit changera également. En outre, les détails de la propriété du capital affectent également les relations de pouvoir qui se produisent dans la prison en tant que champs de combat. Le pouvoir est influencé par le nombre de types de capital possédé et détermine en même temps le type de capital le plus influent pour devenir un personnage puissant en prison, considérant une miniature du monde social.

\section{Discussion}

\subsection{Position initiale de la propriété du capital des personnages dans Un prophète}

L'apparence d'un personnage peut être vue des séquences d'exposition aux celles de dénouement du film. Cependant, certains personnages apparaissent pour la première fois au milieu du film. Les données sur la propriété du capital sont prises depuis la présence de la première personne dans l'histoire. Les informations du capital des personnages se montrent à partir de leur apparence au début de l'histoire dont la base de données se voit comme suit : 
Tableu 1 La propriété initiale des capitaux des personnages

\begin{tabular}{|c|c|c|c|c|c|}
\hline Capital & ue $^{\text {Économiq }}$ & Social & $\begin{array}{l}\text { Cultur } \\
\text { el }\end{array}$ & ue ${ }^{\text {Symboliq }}$ & Remarque \\
\hline Malik & - & - & - & - & $\begin{array}{l}\text { Il n'a ni argent, ni ami, ni } \\
\text { éducation, ni garant }\end{array}$ \\
\hline César & - & $\sqrt{ }$ & $\sqrt{ }$ & $\sqrt{ }$ & Il est chef d'un groupe \\
\hline Réyéb & $\sqrt{ }$ & - & - & - & $\begin{array}{l}\text { Il est trafiquant de drogue en } \\
\text { prison }\end{array}$ \\
\hline Ryad & - & $\sqrt{ }$ & $\sqrt{ }$ & - & Il est marié et bien éduqué \\
\hline Ibanez & - & $\sqrt{ }$ & $\sqrt{ }$ & $\sqrt{ }$ & $\begin{array}{l}\text { Il est gardien de la prison et } \\
\text { proche de César }\end{array}$ \\
\hline Jordi & $\sqrt{ }$ & $\sqrt{ }$ & - & $\sqrt{ }$ & $\begin{array}{l}\text { Il est fournisseur de drogue et } \\
\text { il a des subordonnés }\end{array}$ \\
\hline Moussab & - & $\sqrt{ }$ & $\sqrt{\sqrt{ }}$ & $\sqrt{\sqrt{ }}$ & Il est Imam (chef réligieux) \\
\hline Jacky & $\sqrt{ }$ & $\sqrt{ }$ & $\sqrt{ }$ & $\sqrt{ }$ & Il est chef de la mafia corse \\
\hline
\end{tabular}

Le tableau ci-dessus fournit des informations sur les types de capitaux que chaque personnage d'Un prophète possède. Quatre types de capital selon le concept de Bourdieu sont le capital économique, social, culturel et symbolique (Jourdain \& Naulin, p. 87). En liant la propriété du capital à chaque personnage, on peut voir un capital influent dans une arène. Les personnages énumérés dans le tableau sont des personnalités influentes dans la vie de Malik en prison. Ceux qui sont au-dessus de la double ligne sont des personnages en prison. Les autres sont des personnages qui vivent en dehors de la prison.

Basé sur le tableau 1, Malik est entré en prison sans aucun capital. Des informations sur les capitaux de Malik peuvent être consultées dans la séquence initiale d'Un prophète lorsque Malik a été interrogé pour des procédures administratives visant à être emprisonné. Malik, qui n'a ni argent, ni famille, ni ami, ni garant, ni éducation et ni travail, affaiblit sa position. Cette faiblesse facilite la tâche à d'autres personnages de le saper. Reyeb, qui a un capital économique sous forme de haschich, peut harceler Malik à cause de la position de Malik. César a également utilisé la faiblesse de Malik pour devenir l'exécuteur de la mission qu'il a reçue de Jacky.

César se décrit comme un prisonnier respecté en prison. Il a un grand capital symbolique. Ceci est influencé par le capital social en ayant de nombreux subordonnés et la confiance de Jacky. César est en réalité une extension du chef de groupe corse à l'extérieur de la prison, Jacky Marcaggi. C'est lui qui peut payer et garantir le bien-être du groupe corse. Bien que le capital économique de César ne soit pas apparent, le pouvoir de César est indiqué par son intelligence pour lire la situation et formuler une stratégie pour que personne n'ose le combattre. La proximité de César avec l'un des gardiens, Ibanez, a pu ouvrir l'accès à César pour être plus libre en prison.

Ryad et Jordi aident le faible Malik en prison. Ryad a contribué à donner une éducation et des connaissances à Malik. En tant qu'ami qui est bien éduqué et qui a plus de relations sociales, Malik estime qu'il a besoin d'être protégé par Ryad s'il a des affaires avec un groupe arabe. Ryad avec la plupart des groupes arabes emprisonnés étaient des adeptes de l'Imam Moussab. Ryad a donc pu défendre Malik lorsque le groupe arabe a manifesté une réaction antipathique à Malik. Cela arrive en même façon avec Jordi. Il possède un capital économique sous forme de drogue, car le cannabis peut être considéré comme une nécessité souhaitée par les détenus. Plus un détenu a du cannabis, plus il sera cherché par d'autres détenus. La figure de Jordi est un exemple de propriétaire d'un grand capital économique en prison.

Sur la base des informations expliquées, la description de la propriété du capital de chaque personnage se décrit dans un schéma des positions sociales de Bourdieu. Dans le schéma, il existe deux lignes axiales, à savoir l'axe vertical et horizontal. L'axe vertical du schéma indique le volume global de la propriété des capitaux. L'axe horizontal est une opposition aux variations entre le capital économique et le capital culturel (Jourdain \& Naulin, pp. 94-95). Si la description de la propriété des capitaux dans les personnages du film est mise en œuvre dans le schéma des positions sociales de Bourdieu, la représentation graphique suivante est obtenue : 


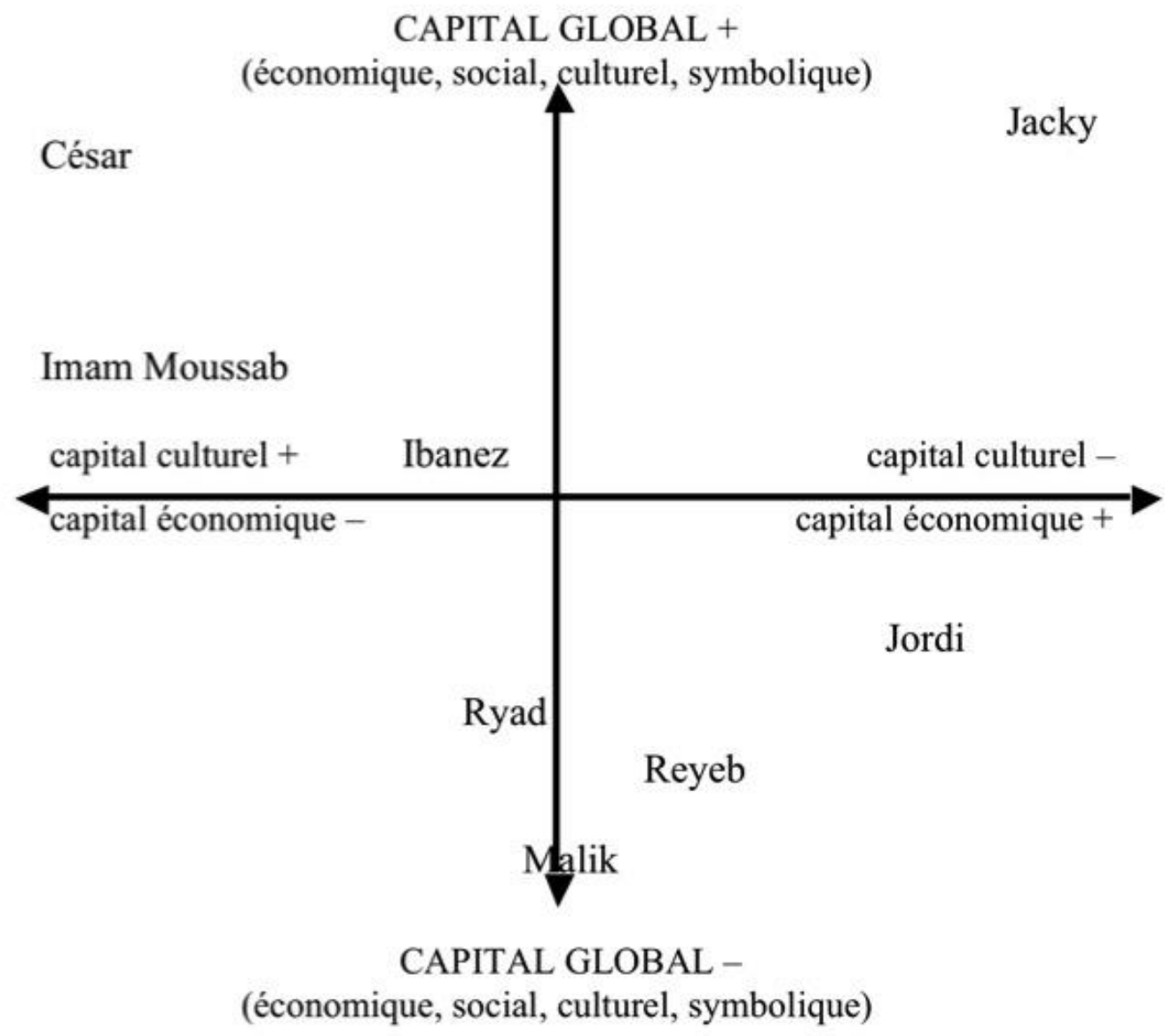

Figure 1 Espace initial des positions sociales des personnages d'Un prophète

$\mathrm{Au}$ bas de l'axe horizontal, Jordi a assez de cannabis, il est donc mis à droite car on considère la drogue comme un capital économique. La position de Malik au bas de la cartographie a été causée par son manque de capital. A l'inverse, les personnages au-dessus de la ligne horizontale ont de grands volumes de capital, tels que le grand nombre de subordonnés pour César, les adeptes nombreux pour l'Imam Moussab, la position d'Ibanez à cause de son pouvoir à la prison, et Jacky en tant que chef de mafia corse qui est très riche. C'est la raison pour laquelle sa position est la plus haute du schéma. La position de César en tant que confident de Jacky a rendu la position de César plus élevée que celle d'autres prisonniers.

La haute position du pouvoir de César était basée sur le capital symbolique visible de ses subordonnés et selon les ordres de César. Le prestige de César se faisait sentir en marchant avec eux. Le capital social de César était très élevé grâce à Ibanez qui devient son complice. En plus, il était également proche du plus haut dirigeant du groupe corse, Jacky. Le capital culturel de César semble également élevé lorsque l'on considère des stratégies qui donnent l'impression que César est une personne intelligente pour planifier une mission.

\subsection{Mouvement de la position de Malik et de César}

Au fur et à mesure de l'histoire d'Un prophète, il y a des mouvements de capitaux qui se produisent dans deux personnages importants, à savoir Malik et César. D'autres personnages n'ont pas de grand changement, car ils n'ont pas de tendance à se battre pour défendre ou collecter les capitaux. Ils exercent encore leurs rôles et fonctions comme au début de l'histoire. Cependant, plusieurs personnages supplémentaires ont influencé le mouvement des capitaux de Malik et de César. 
Tableu 2 Le changement des capitaux des personnages

\begin{tabular}{|l|c|c|c|c|c|}
\hline Capital & Économiq & Socia & Cultur & Symboliq & Remarque \\
\hline Malik & $\sqrt{ }$ & $\sqrt{ }$ & - & - & $\begin{array}{c}\text { - il a commencé à avoir un revenu } \\
\text { sous forme de drogue et d'argent } \\
- \text { il a commencé à se faire des } \\
\text { amis avec plusieurs détenus }\end{array}$ \\
\hline César & - & $\sqrt{ }$ & $\sqrt{ }$ & $\sqrt{ }$ & $\begin{array}{c}\text { Il a commencé à perdre ses } \\
\text { subordonnés mais il a toujours } \\
\text { Malik comme complice }\end{array}$ \\
\hline Reyeb & - & - & $\sqrt{ }$ & $\sqrt{ }$ & $\begin{array}{l}\text { Il est une projection de la } \\
\text { culpabilité et de la vengeance de } \\
\text { Malik }\end{array}$ \\
\hline
\end{tabular}

Selon le tableau 2, Malik a appris un développement de capital depuis qu'il a reçu l'autorisation de sortir de la prison pour une journée. Cette séquence montre la première tentative de Malik pour obtenir des capitaux. Cette première tentative a commencé par atteindre le capital économique, se présentant sous la forme de 5000 euros, qu'il a mérités comme honoraires du groupe corse, et de 25 kilos de haschich grâce aux informations de Jordi.

On voit le rôle de Jordi pour Malik, à savoir comme un assistant pour obtenir un capital économique. Ryad joue aussi en tant que complice de Malik en dehors de la prison. La fidélité de Ryad a aidé Malik à poursuivre le capital économique qu'il en avait besoin. En revanche, Malik utilise le capital symbolique de Jordi en tant que distributeur de haschich en prison et Ryad en tant que distributeur en dehors de la prison, considérant que Malik en prison ne peut échapper à l'influence de César. Malik a essayé de se cacher de César en ce qui concerne les affaires avec Jordi et Ryad. Il n'a que du capital économique et du capital social en ce moment. Cette acquisition de capital n'est toujours pas suffisante pour lutter contre César. Bien que César n'ait aucun capital économique, son capital social et symbolique est encore plus élevé. César reste au pouvoir parce que son président en dehors de la prison est le propriétaire du plus grand capital économique.

César était un autre personnage qui a connu des mouvements de capitaux. Ceci est vu explicitement dans la séquence de la libération des subordonnés de César. Cela réduit directement le capital social de César en prison. César, sans les subordonnés, a également réduit le capital symbolique de César en tant que dirigeant en prison. Cependant, le rôle de César n'a pas complètement disparu. César a toujours Malik en prison, alors Malik peut encore être l'exécuteur des souhaits de César. En outre, César était toujours proche d'Ibanez, le gardien sous l'influence de César.

En examinant les mouvements de capitaux, Malik et César ont appris des mouvements importants. Cependant, le mouvement de Malik ne suffisait pas à le faire avoir un grand pouvoir en prison. L'augmentation de Malik n'est que dans le capital économique. César a toujours les mêmes capitaux, mais leur volume diminue. Si Malik veut gouverner en prison, il doit gagner la position de César au début de l'histoire. Ainsi, la personne qui a le capital le plus élevé est toujours Jacky, de sorte que la chute de César est toujours protégée par Jacky. Les changements sur le capital de Malik et de César peuvent être compris dans le schéma suivant : 
CAPITAL GLOBAL +

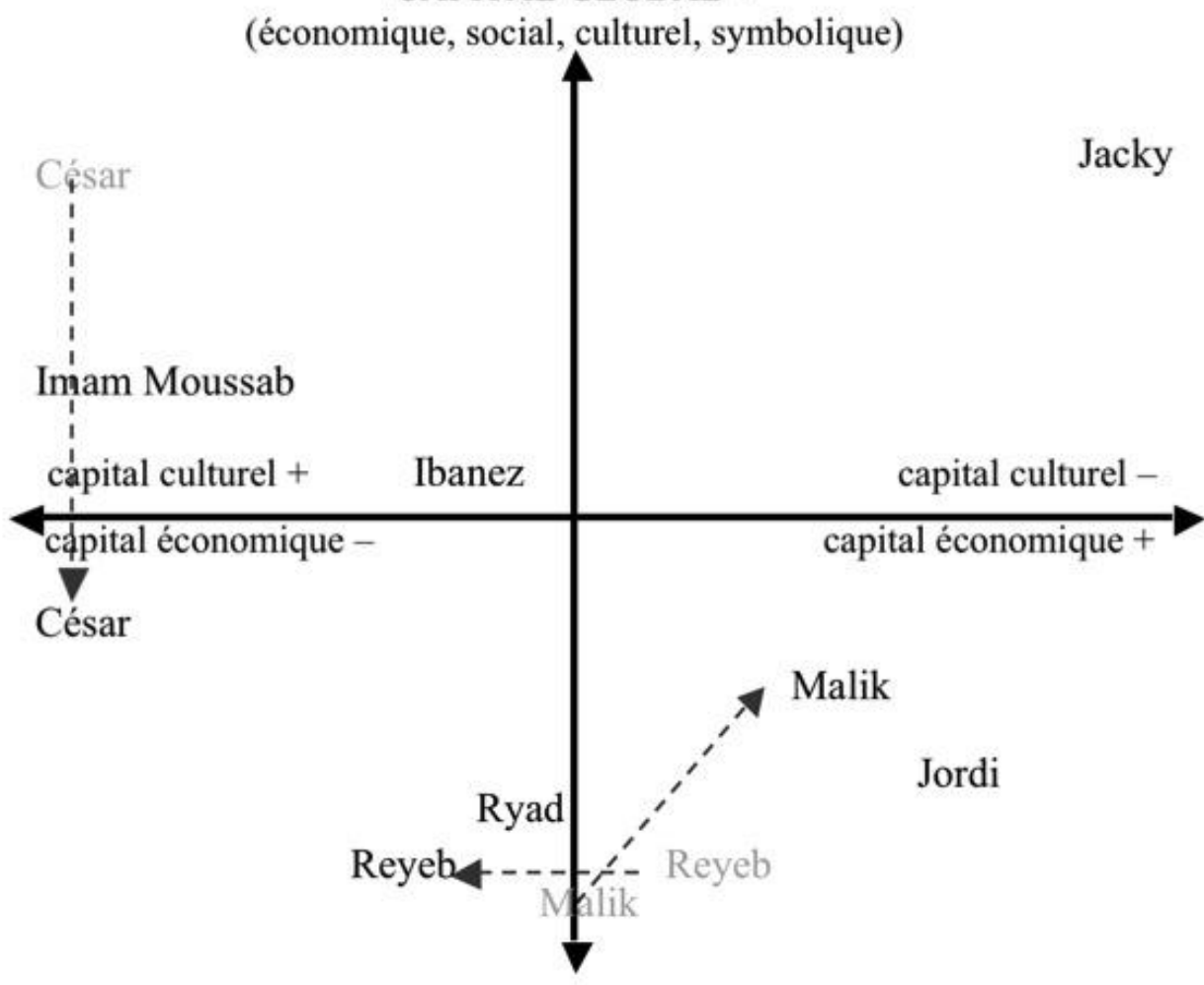

CAPITAL GLOBAL -

(économique, social, culturel, symbolique)

Figure 2 Le mouvement des positions sociales des personnages d'Un prophète

Sur la base des informations présentées dans l'image 2, les mouvements de position se produisent uniquement à Malik, César et Reyeb. Malik était plus grand que Ryad et Jordi parce que Malik était un seul complice direct de César puisque la plupart des membres du groupe corse avaient été libérés et que Malik était capable de les commander sur le trafic de drogue. Une chose différente est arrivée à l'apparition du personnage de Reyeb. Cette figure réapparaît comme l'imagination de Malik, de sorte que le capital qu'il possède semble différent de son apparence humaine au début de l'histoire. Reyeb était une image de culpabilité et de ressentiment de Malik étant donné la pression du pouvoir de César.

Comme Reyeb avait été tué au début de l'histoire, il n'y avait pas beaucoup de capital que Reyeb pouvait posséder. À la fin de l'apparition de Reyeb, il a interprété la danse soufie et lu une des lettres coraniques qui fournissait des informations sur le capital culturel de Reyeb. La position de Reyeb n'a changé que parce que le type de capital possédé est devenu différent, mais la figure de Reyeb avec son capital ne pouvait être ressenti que par Malik. La position de Reyeb est la plus basse du schéma.

\subsection{Position finale de Malik et de César}

Le mouvement de la position de capitaux de Malik et César se poursuit jusqu'à la fin de l'histoire. En outre, Ibanez a également connu des mouvements de capitaux dès que le déclin du capital de César. La différence entre les mouvements graphiques de Malik et de César est liée à leur stratégie d'acquisition du capital et à leur défense du capital. En fin de compte, la personne qui gagne la plus haute position aura le pouvoir en prison. Plusieurs personnages apparaissent sous la forme finale de la propriété du capital, à savoir Hassan et Brahim. Les deux affectent l'acquisition du capital de Malik. 
Tableu 3 La propriété finale des capitaux des personnages

\begin{tabular}{|c|c|c|c|c|c|}
\hline Capital & ue $^{\text {Économiq }}$ & Social & el Cultur & ue ${ }^{\text {Symboliq }}$ & Remarque \\
\hline Malik & - & $\sqrt{ }$ & $\sqrt{ }$ & $\sqrt{ }$ & $\begin{array}{l}\text { Il a donné tout l'argent généré } \\
\text { pour l'imam moussab jusqu'à ce } \\
\text { qu'il soit respecté par les Arabes }\end{array}$ \\
\hline César & - & - & - & - & $\begin{array}{ccc}\text { Il a perdu } & \text { tous les } \\
\text { subordonnés et } & \text { toute } & \text { la } \\
\text { confiance de Jacky } & & \end{array}$ \\
\hline Ibanez & - & - & - & - & $\begin{array}{l}\text { Il a été renvoyé en tant que } \\
\text { gardien de prison }\end{array}$ \\
\hline Hassan & - & $\sqrt{ }$ & $\sqrt{ }$ & - & $\begin{array}{l}\text { Il a beaucoup de réseaux aux } \\
\text { communautés arabes }\end{array}$ \\
\hline Brahim & $\sqrt{ }$ & $\sqrt{ }$ & - & $\sqrt{ }$ & Il est chef de la mafia arabe \\
\hline
\end{tabular}

Dans le schéma précédent, le mouvement de Malik a commencé quand il avait un capital économique abondant. Bien que César ne semble pas avoir de capital économique, il est protégé par son capital social en ayant Jacky comme son patron. Finalement, Malik a de la chance pour changer la position lorsque César lui a ordonné de tuer Jacky. Malik a compris que Jacky a réussi à renverser le capital de César. Par conséquent, au lieu de tuer Jacky, Malik a opposé Jacky et César pour que le capital social de César soit perdu.

Malik a mis au point une autre stratégie, en passant par la recherche d'un capital social plus élevé. Lorsque Malik a trouvé un emploi chez César pour se rendre à Marseille, Malik a rencontré Brahim Lattrache. Brahim pensait que Malik avait des capacités spéciales. Malik a profité de cette occasion pour approcher Brahim afin qu'il soit invité à travailler avec lui en dehors de la mission de César. Le capital social de Malik s'est également développé dans le groupe arabe à Marseille.

L'aide de Brahim a permis à Malik d'approcher Latif pour développer le commerce de la drogue. Avec un capital économique sous forme d'argent provenant de la vente de drogue, Malik l'a donné à l'Imam Moussab pour qu'il puisse approcher le groupe arabe de la prison qui était de fidèles partisans de l'Imam. Hassan a été offensé au début parce que Malik a donné de l'argent illicite à l'Imam Moussab. Cependant, grâce à l'intelligence de Malik, il a pu négocier avec Hassan et Malik a finalement obtenu le cœur du groupe arabe.

La stratégie de Malik a réussi à détruire César. Ibanez a été arrêté alors il n'était plus gardien de prison. César, qui n'a plus la confiance de Jacky et qui n'a plus de subordonnés, a perdu ses capitaux. Indirectement, le capital symbolique en tant que chef de groupe a disparu. Au contraire, Malik possède un capital social élevé et est donc protégé par des groupes arabes. La position de Malik et de César en fonction de leur propriété des capitaux se montre dans le tableau suivant : 


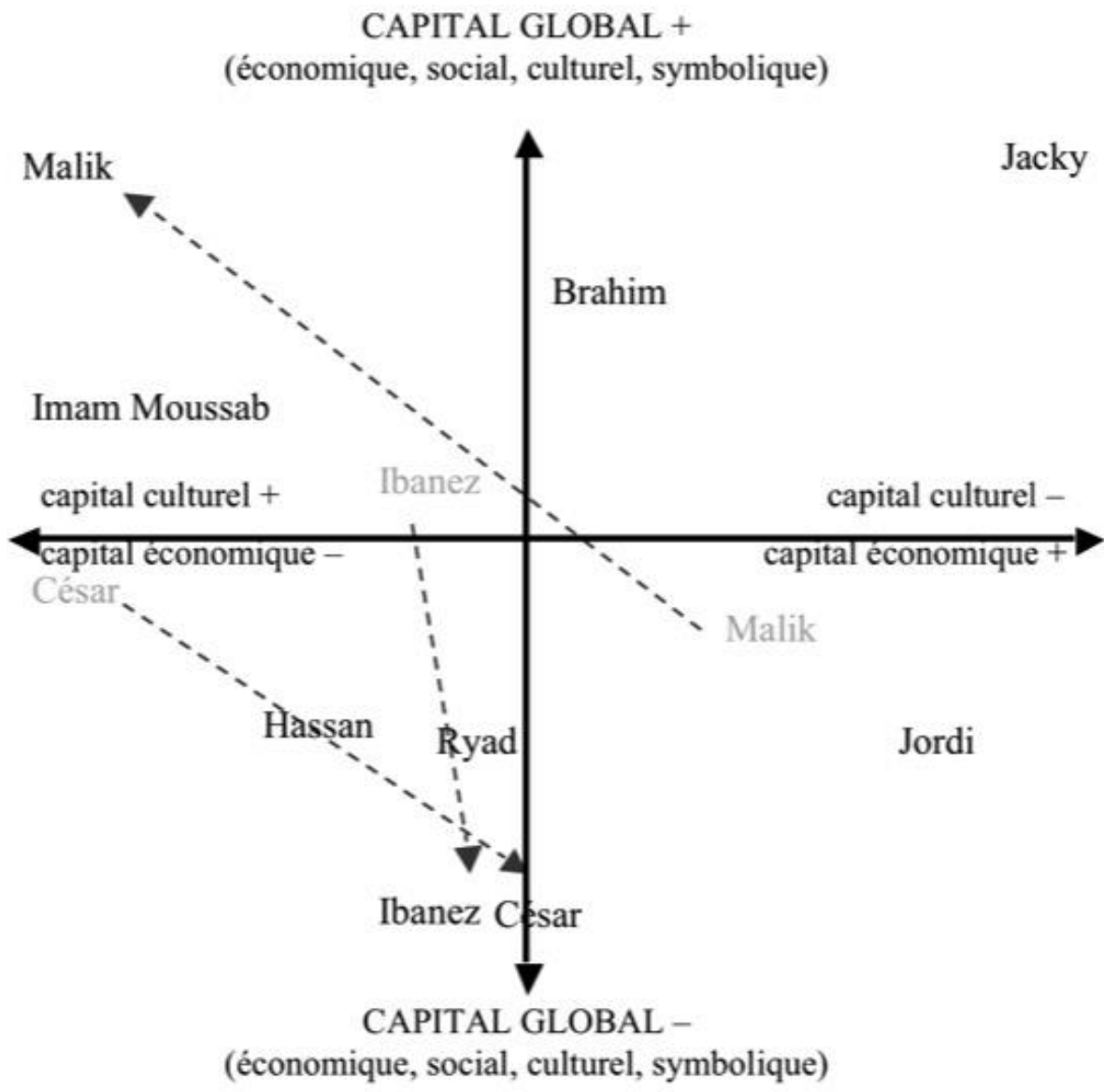

Figure 3 Espace final des positions sociales des personnages d'Un prophète

Le schéma ci-dessus montre l'augmentation rapide du capital de Malik. Grâce au rêve de Malik qui semblait voir un événement futur, la vision de Malik devient le capital symbolique de Malik à la focalisation de Brahim. Au lieu d'obtenir un grand capital économique, Malik a fourni toute la richesse de la vente de drogue pour augmenter le capital social. La stratégie de Malik visant à accroître le capital social pour lutter contre César est le fruit du capital culturel de Malik, qui lui donne l'intelligence d'étudier la situation en prison. En fin de compte, Malik a réussi à changer la position du pouvoir en prison. Comme Malik était en prison, il a pu se renseigner sur l'habitus du groupe arabe qui respectait l'Imam Moussab. Malik utilise la figure de l'Imam Moussab pour attirer l'attention des groupes arabes en prison.

\section{Conclusion}

La propriété du capital est une référence pour les luttes de pouvoir parmi les personnages dans $U n$ prophète. Parmi les trois schémas de capital décrits, Malik a conçu une stratégie pour se libérer de la domination de César. Une façon de réaliser son désir est de vaincre le pouvoir de César dans le champ de prison. Les efforts de Malik pour entrer dans le groupe arabe ont aidé à se développer au champ de prison.

On a étudié la structure de la propriété du capital dans les prisons. Malik a indirectement enregistré l'habitus de la domination César, y compris les moyens de devenir un leader et de gérer la stratégie d'une mission. Comme le capital culturel de Malik augmente avec l'éducation en prison et en apprenant de sa situation de vie déprimée, il commence par augmenter le capital économique. La variation et la quantité de capital économique en prison ne changent pas la position de Malik avec César. Malik est respecté par nombreuses personnes grâce à son capital symbolique en tant que fournisseur de drogue.

Avec de grandes quantités de capital économique, Malik l'accumule avec le capital social. Malik a cherché des leaders influents dans d'autres groupes pour que Malik puisse augmenter le capital social. Après avoir eu le grand capital social, la stratégie finale de Malik consistait à attendre une opportunité qui pourrait faire tomber César. Avec ce changement de position de pouvoir, César était en position de Malik au début de l'histoire. Inversement, Malik semblait remplacer César en prison. 
La prison dans Un prophète est une représentation du petit monde avec l'arène des groupes sociaux qui ont un chef comme un syndicat. Dans ce domaine des combats de groupes sociaux, la propriété du capital social est très importante pour faire tomber d'autres groupes. En considérant Malik comme un agent dynamique, la démarche visant à obtenir un capital social commence par l'enrichissement du capital culturel pour obtenir un capital économique pouvant être utilisé pour gagner la loyauté des gens. De cette façon, il gagnera un capital social absolu. La stratégie utilisée par Malik est une approche des agents qui ont un capital social étendu. Un capital social solide peut être utilisé pour lutter contre le capital symbolique construit depuis longtemps.

\section{Références}

Audiard, J. (2009). Un prophète. France: Why Not Productions: UGC Vidéos.

Aumont, J., \& Michel, M. (2008). L'Analyse des Films (2nd ed.). Paris: Armand Colin.

Boggs, J. M., \& Petrie, D. W. (2008). The Art of Watching Films (7th ed.). New York : McGraw Hill.

Bourdieu, P. (2001). Langage et pouvoir symbolique. Paris : Éditions Points.

Haryatmoko. (2010). Dominasi Penuh Muslihat: Akar Kekerasan dan Diskriminasi. Jakarta : Gramedia

Pustaka Utama.

Jourdain, A., \& Naulin, S. (2011). Sociologies Contemporaines : La Théorie de Pierre Bourdieu et Ses

Usages Sociologiques. Paris : Armand Colin.

Widjojo, M. (2002). Strukturalisme konstruktivis: Pierre Bourdieu dan Kajian Sosial Budaya. In S. Irzanti

\& A. A. Harapan (Eds.), Prancis dan Kita. Jakarta: Wedatama Widya Sastra. 